\title{
Papyrus und Lexikon.
}

\section{Wörterbuch und Wortverzeichnis.}

Wenn in der Sprache $\varphi v \sigma_{\iota}$ und $\psi v \chi \eta^{\prime}$, Physiologie und Psychologie sich berühren, so bildet die Sprachwissenschaft den Übergang von den Naturwissenschaften zu den humanistischen. Während nun die Grammatik in einer durch die Jahrhunderte sich hindurchziehenden Folge wie eine exakte Wissenschaft bearbeitet wurde, fafste man die Lexikographie bis in die Mitte des ablaufenden Jahrhunderts meist als Kunst oder als Kunstgewerbe auf. In der That bietet dieser Zweig der Forschung so verschiedene Seiten, dafs ein methodisches Vorgehen hier die Voraussetzung wohl völligen, aber nicht jedes Erfolges war

Gegenwärtig sind grofse Gegensätze herausgearbeitet, und es sind mannigfache $\mathrm{Z}$ wecke gesondert, denen ein Stellenverzeichnis und, was darauf gebaut wird, zu dienen hat. Die Semasiologie, die Entwickelung und das Leben des Wortes in der Geschichte, ist dem allgemeinen Wörterbuch zu überweisen; Speziallexika geben ein Inventar des Wortschatzes bei einem Autor oder einer Klasse von Autoren und weisen den diesem Autor eigentümlichen Gebrauch namentlich durch Herzählung der Verbindungen nach (Objekte beim Verbum, Substantive beim Adjektivum u. s. w.), in denen bei ihm das Wort sich findet. Voll-

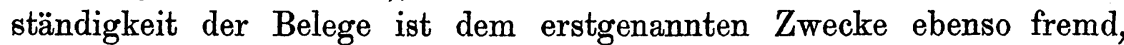
wie dem letzteren natürlich; und so steckt in dem Spezialwörterbuch auch ein verbaliter geordnetes Reallexikon: für ein Reallexikon aber ist die Einteilung nach Verbindungen hemmend, und so behauptet gegenüber dem Wörterbuch das einfache Stellenverzeichnis seinen Platz welches allerdings dem Benutzer die Mühe auferlegt, das von ihm Gesuchte selbst auszusondern, aber auf der andern Seite es ihm erspart, eine nicht im Zuge seiner Wünsche liegende Ordnung wieder aufzulösen. Es mag in manchen Fällen zweifelhaft sein, ob Stellenverzeichnis oder Wörterbuch wichtigeren Interessen dient; in andern Fällen mag eine beispielweise angeführte Gliederung auch nur einiger Worte ein wertvolles Hilfsmittel für die Benutzung eines Wortverzeichnisses sein, - für ein Material, das erst gewonnen und stetig vermehrt wird, wie 
die Papyri, sind nur Stellenverzeichnisse am Platze ${ }^{1}$ ), daneben eine Übersetzung der Worte, die im Griechisch der Papyri etwas anderes bedeuten, als im Attischen. Kommt es einmal zu einem Corpus Papyrorum, so wird für die Urkunden und Briefe, für alle nicht litterarischen Papyri, die Frage nach der Beschaffenheit des lexikographischen Appendix keine der unwichtigsten sein.

\section{Die provisorischen Indices der Sonderpublikationen.}

Das gegenwärtige Stadium der Papyruskunde bietet Editionen, die, von den verschiedensten Seiten ausgehend, der Zentralstelle entbehren; doch sind die Indices im wesentlichen nach einem Schema entworfen, das demjenigen der epigraphischen Arbeiten entspricht: Prosopographia, Realia, Index potiorum verborum; nur ist der letztere bei den Papyri auf mehr Worte ausgedehnt.

Betrachten wir zunächst den Wortindex, so ist der Name GeneralIndex, den er bei Grenfell und Hunt (Oxyrhynchos I) hat, der sachgemälse; nur mülste diesem Namen auch die That entsprechen, und die verhältnismärsig wenigen kleingeschriebenen Worte, die in den Realindices stehen (Menschen- und Ortsnamen natürlich nicht), in diesem GeneralIndex, mindestens als Lemmata, mit Verweisung wiederholt werden; denn die Grenze für das, was noch in die Specialia gehört, ist nicht mit Sicherheit zu ziehen, und der Leser, der $\dot{\eta} \gamma \varepsilon \mu$ sov $v^{\prime} \omega \dot{\eta} \gamma \varepsilon \mu o v i \alpha$ sieht, und $\dot{\eta} \gamma \varepsilon-$ $\mu \omega ́ \nu$ vermilst, stutzt ebenso wie der, welcher (Oxy. I) $\dot{\varepsilon} \mu \beta 0 \lambda \dot{\eta}$ nicht findet, sondern letzteres unter X: Taxes suchen soll, während $\dot{\varepsilon} \mu \beta o \lambda \alpha^{-}$ $\tau \omega \varrho$ unter VIII: Officials steht. Im ersten Beispiel kann man noch einwenden, dafs der Beamten-Index bekannt ist, im zweiten ist die

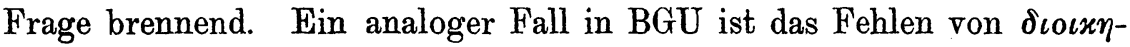

1) Es mag die Bemerkung nicht überflüssig sein, dafs ein Stellenverzeichnis nicht nur dazu benutzt werden kann, das Vorkommen des einzelnen Wortes zu konstatieren, sondern in der einfachsten Weise für Wortverbindungen benutzbar wird, wenn man, am besten mit zwei Exemplaren des Verzeichnisses arbeitend, die Zahlen der Stellen des einen Wortes und die der Stellen des anderen vergleicht und, wo beide zusammentreffen, auf die Verbindung schliefst. Will man z. B. in

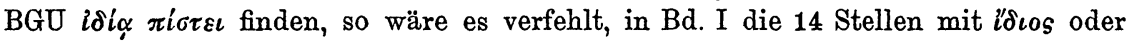

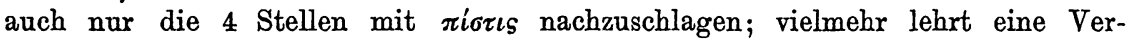
gleichung der Nummern von i'sıos mit denen von $\pi i \sigma \tau \iota s$, dafs ein und dieselbe bei

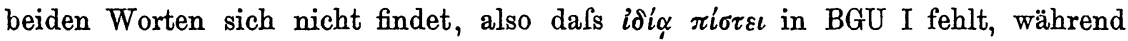
umgekehrt in BGU II der Index das Vorkommen dieser Verbindung nur für 371, 19

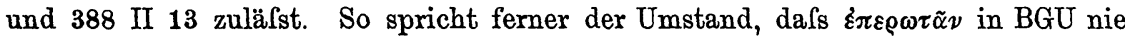

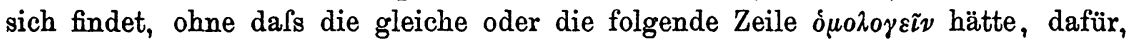
dafs $\dot{\varepsilon} \pi \varepsilon \rho \omega \tau \tilde{\alpha} \nu$ nur in der Formel: $\varepsilon \pi \varepsilon \rho \omega \tau \eta \vartheta \varepsilon i s ~ \dot{\omega} \mu 0 \lambda o ́ \gamma \eta \sigma \alpha$ auftritt; indefs ist hier Vorsicht am Platze. 


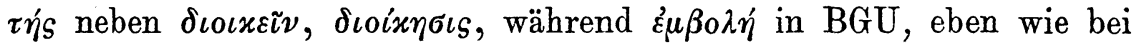
Kenyon, mit Recht im Wort-Index steht.

Erwägt man, dals $\dot{\varepsilon} \mu \beta 0 \lambda \eta^{\prime}$ im General-Index Oxy. nicht steht, in den beiden andern doch, so empfindet man die Gefahr, dals der Benutzer wähnt, das Wort fehle in Oxy. Daraus ergiebt sich einmal, dafs, wie bemerkt, die Beamten zu rekapitulieren sind, und sodann, dafs Gleichmälsigkeit bei der Herstellung erforderlich ist. Gewifs sind die Einzelpublikationen der Gegenwart, von der Seite des Herausgebers betrachtet, selbständige Werke, deren Autoren auf eigene Verantwortung und also nach eigenen Regeln vorgehen. Der eine mag Übersetzungen geben, der andere Paraphrasen, der dritte Inhaltübersichten, der vierte blos den Text; der eine mag drucken, der andere autographieren, der eine accentuieren und interpungieren, der andere nicht; der eine mag sachlich ordnen, der andere zeitlich, der dritte gar nicht; hier sind die Abweichungen erträglich, weil die Hauptsache, die Urkunde, unter allen Umständen geboten wird, und superflua non nocent; aber für den Benutzer sind diese Werke verschiedener Autoren nur Teile eines Ganzen, das stets anwächst, aber soweit es erschienen ist, vollständig von ihm. verwertet werden soll, und darum wäre es gerade bei den Indices von eminentem Wert, wenn die Verfasser hier eine Verständigung unter sich erzielten, nach der die überhaupt auszulassenden $W_{0 r t e}{ }^{1}$ ) ein für alle mal gleichmäfsig festgestellt und bekannt gegeben würden (prohibentis melior condicio käme hier dem omittere prohibenti zu), und ebenso das Gerüst der ganzen Indicistik festgelegt würde. Denn ein Index ist ein wichtiges, aber auch ein schwerfälliges Werkzeug, und was geschehen kann, ihn handlich zu machen, das sollte nicht unterbleiben.

Ganz äufserlich, aber ebenso schwerwiegend ist folgender Punkt: soll nach Urkunden oder nach Seiten zitiert werden? Wenn Kenyon nach Seiten zitiert, so ist dies nur aus dem Fehlen laufender Nummern bei der Edition des British Museum zu erklären; es wäre dringend zu wünschen, dafs in weiteren Bänden (wie die Berliner Publikationen dies thun) neben der Ordnungsnummer des Museums noch die laufenden Nummern der Publikationen, und zwar in arabischen Ziffern ${ }^{2}$ ) eingeschaltet würden. Der vortreffliche Index zu Kenyons Publikation hat

1) Die Kenyon also zusammenfafst: the commonest words. - Es ist nicht $\mathrm{zu}$

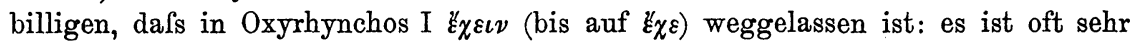
wichtig zu erwägen, welches Objekt bei $z^{\prime} \chi \varepsilon \iota \nu$ in einer Lücke gestanden haben

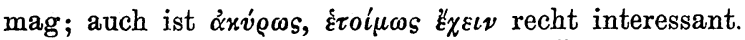

2) Oxy. I hat lateinische, was der Übersicht so sehr Abbruch thut, dals sie im Index durch arabische ersetzt sind. 
dadurch, dals er es verschmäht hat, neben die Zahl der Seite noch die Zeile des Papyrus zu setzen, seine Brauchbarkeit vermindert, es würde sich wohl lohnen, ihn nochmals abzudrucken, und hinter die Seitenzahl die Ordnungs-Nummer und die Zeilenzahl des Papyrus zu setzen, z. B.

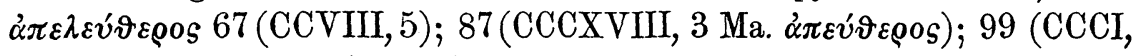
$5)$, oder, besser: $67(208,5)$.

Dagegen sollte allgemein angenommen werden die Anstirnung der den gangbaren Wörterbüchern fremden Wörter, wie sie sich bei Kenyon findet; sie gewährt eine bequeme Übersicht über die neuen Wörter in dem Griechisch der Papyri.

Auch für die Aufzählung der Stellen mufs davor gewarnt werden, um der Raumersparnis willen die Brauchbarkeit zu schmälern. Man kann sagen, dals es minder beschwerlich ist, sogar eine grölsere Zahl von Stellen im Zuge des Buches nachzuschlagen, als eine kleinere im Hinund Herblättern, geschweige denn eine gleiche; und es ist nicht nur weit vorzuziehen, wenn verdienstliche Bemerkungen, wie die Kenyons über sonderbare Formen, an die betreffende Stelle im Zuge der Nummern hinter der Zahl in Klammern beigefügt werden, statt dafs jetzt die Stellen am Schlufs zusammengesetzt werden ${ }^{1}$ ), sondern es sollte in solchen Fällen, wo durch die Einordnung derartiger Bemerkungen häufige Wiederholung sich notwendig machen würde, zunächst eine Übersicht aller Stellen gegeben werden, und die Stellen, für welche die Bemerkungen gelten, nachher, etwa durch: $-N$. B. getrennt wiederholt werden, wie denn überhaupt die wiederholte Aufführung einiger Zahlen wenig Raum mehr fordert und sehr viel Zeit ersparen kann.

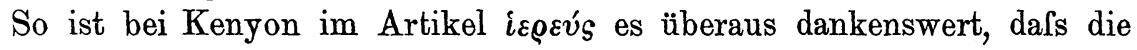
Dienstherren adnotiert werden, aber es sollten darum, dafs die Stellen, in welchen diese Heiligkeiten genannt sind, am Schlusse gesondert angeführt werden, diese Stellen in der Aufzählung am Anfang nicht fehlen. $\left.{ }^{2}\right)$ Es würde in diesem Falle demjenigen, dessen Interesse sich nicht auf das Heiligtum, sondern auf die Stellung des Priesters als solchen (etwa bei Rechtsgeschäften) richtet, es erspart bleiben, nach

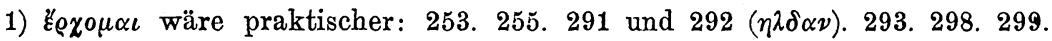
301 ( $\varepsilon \alpha \nu \varepsilon \lambda \delta o v \sigma \iota) .303$ etc.; statt dals jetzt $\eta \lambda \vartheta \alpha \nu$ 291. $292, \varepsilon \alpha \nu \varepsilon \lambda \delta o v \sigma \iota 301 \mathrm{am}$. Schlufs steht, und also der auf Formenlehre nicht ausgehende Benutzer im Text zurückblättern mufs. - Ebenso wäre bei $\dot{\eta} \mu \varepsilon \rho \rho \alpha$ einfacher: 290. 299. 299 ( $\alpha v \tau \varepsilon$ $\eta \mu \varepsilon \rho \varepsilon)$. 300. - Bei हैं $\varepsilon £ \rho$ s käme besser zu Anfang, statt hinter allen Stellen: 96 $(\gamma \eta \varepsilon \tau \varepsilon \rho \alpha)$ 104. - In gleicher Weise liefsen sich die Adjektiva anhängen bei

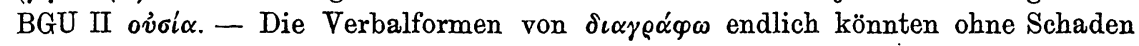
fortbleiben.

3) Es sind: 34. 34. 68. 112. 151. 170. 201. 202. 211. 221; im ganzen würde ihre Aufführung in der Reihe noch nicht eine Zeile ausmachen. 
durchblättertem Buche nochmals hin und wieder zu suchen. ${ }^{1}$ ) Ebenso wäre in dem Artikel $\lambda$ ó yos BGU II zunächst eine Übersicht aller Stellen zu geben (was 7 Zeilen mehr machen würde) und dann allenfalls die notabilia zu bringen, deren Notabilität allerdings nur subjektiv ge-

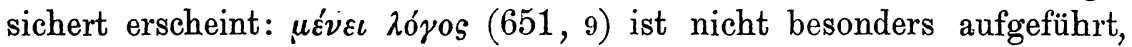

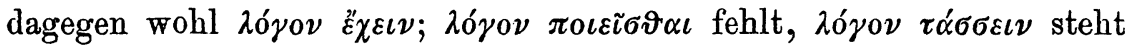

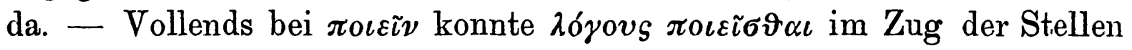

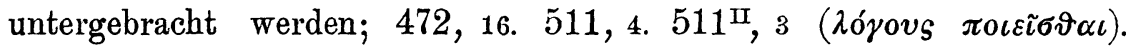
538,17 u. s. f., indem eben die Regel ist, dafs die hinter der Zahl vor dem Punkt stehende Klammer Worte einschliefst, die sich nur auf die eine Stelle beziehen, während Rubriken, die für mehrere Stellen gelten, ohne Klammer, mit einem Doppelpunkt hinter sich, vor der Stelle stehen. Bei $\pi \lambda \eta \dot{\rho} \eta s(\mathrm{BGU})$ ist die Sonderung in $\pi \lambda \eta \dot{\rho} \eta s, \varepsilon i s \pi \lambda \eta \eta_{\zeta}$, $\xi x \pi \lambda \eta^{\prime} \varrho v_{s}$ unnötig, und insofern irreleitend, als die andern Stellen ebenfalls den Zusammenhang haben, in dem die Formel $\dot{\xi} x \pi \lambda \eta_{\text {joovs }}$ als die korrekte erscheint, nämlich die völlige Befriedigung. ${ }^{2}$ )

Eine grofse Erleichterung könnte im Anschlufs an ein Verfahren gegeben werden, das Wilcken in seiner sachlichen Übersicht über alle Urkunden von BGU II befolgt: er druckt die Urkunden von Diokletian an kursiv. Der Benutzer, der nur auf den Prinzipat es abgesehen hat, wird angenehm empfinden, wenn das Nachschlagen der byzantinischen und arabischen Urkunden ihm erspart bleibt, und umgekehrt. Will BGU auch für den Index diese Erleichterung bieten, so mufs es auch da kursive Ziffern wählen, während die nach Zeiträumen geordneten englischen Publikationen einfach durch Querstriche im Index andeuten könnten, wo die neue Gruppe anfängt. Kenyons Publikation hätte vier Gruppen: 1. Ptolemaic Periode. - 2. Roman Periode. - 3. Byzantine Periode. 4 th. century. - 4. Late Byzantine papyri. Indefs brauchten 1 und 2 nicht getrennt zu werden, und z. B. der Artikel $\delta i \delta \omega \mu \iota$ könnte lauten: $\delta i \delta \omega \mu \iota ~ 3.99 .153$ (u. s. f.) 251. 252 . | 290 (u. s. f.) 319 . \| 326 u. s. f.

Die Dreiteilung der englischen Publikationen verdient $\mathrm{m}$. E. den Vorzug vor der Zweiteilung; nur ist es besser, mit Wilcken die mittlere Periode schon mit Diokletian beginnen zu lassen: sie ist ohnehin die schmalste.

1) Man halte diese Bemerkung nicht für Pedanterie; es ist nicht blos die Zeitversäumnis, die man empfindet, wenn man zurückblättern mufs, sondern es richtet sich die Aufmerksamkeit gewissermalsen auf das ganze Buch ein, und hat man es durch und muls wieder anfangen, so erfordert das eine neue Anspannung.

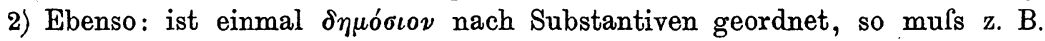

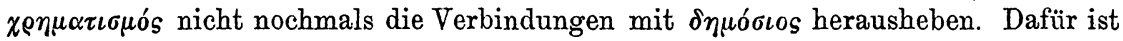

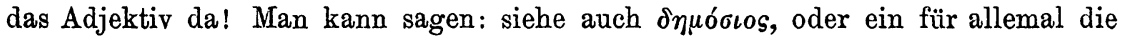
vorkommenden Adjektiva in Klammern dem Lemma beifügen. 
In einem andern Sinne noch können die Indices ein Bindeglied zwischen den einzelnen Publikationen werden: die Papyri bieten in dem Teile der Masse, der die Urkunden umfafst, hunderte von similia; die Ergänzung und selbst die Lesung der nova wird vielfach gefördert durch die Erinnerung an Bekanntes und die Vergleichung mit diesem. Nun ist es allerdings nicht eine unerschwingliche Arbeit, in den nach sachlichen Gesichtspunkten geordneten Sammlungen alle verwandten Stücke durchzusehen, und wer ein oder zwei Exemplare der ungeordneten Publikationen daran wenden will, kann sich auch diese zum gleichen Zweck zurechtschneiden, - aber dieser Weg ist nicht angenehm und führt sicher nicht zum Ziel. Viel besser ist es, die auffallenden, kritischen Worte des novum im Index nachzuschlagen und also die similia aufzuspüren.

So hat sich Kenyon beim Papyrus CCXCIII (Brit. Mus. II pag. 187/8) die Mühe nicht verdriefsen lassen, das Corpus Papyrorum Raineri nach analogen Urkunden durchzusehen und dabei No. XI gefunden, die ihm wahrscheinlich für die Ergänzung wertvoll war. - Nehmen wir aber den Weg durch den Index, so ist es leicht, von dem, was selbst ein Entzifferer wie Kenyon nicht gefunden, noch einiges aufzuspüren. Wir müssen zunächst fragen, welches die dem vorliegenden Papyrus eigentümlichen, seltenen Wendungen sind: hier fallen von juristischen Ter-

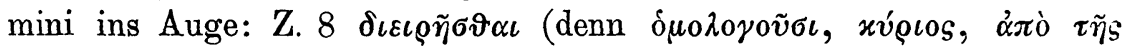

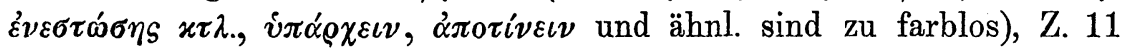

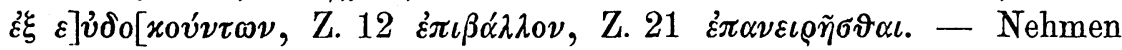
wir hiervon $\left.\mathfrak{z}^{\xi} \varepsilon\right] \hat{v} \delta o[\varkappa o v ́ \nu \tau \omega \nu]$, so ergiebt der Berliner Index bei $\varepsilon \dot{v} \delta o-$ xєĩv 96,16 und öfter, 101, 23. 193, 25. 300, 11. 427, 21. 444, 8. 543, 15. Von diesen bieten $96,101,193,300$ die gewöhnliche Ratihabition, 427 und 543 das Versprechen, sie herbeiführen zu wollen, aber 444, 8 zeigt:

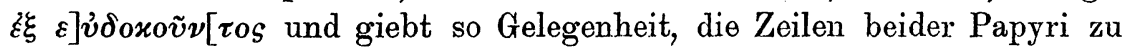
verbessern. ${ }^{1}$ ) BGU 444 zeigt sich bald als ähnliche Teilungsurkunde mit $\delta \iota \alpha i \varrho \varepsilon \sigma \iota \varsigma$ und $\varepsilon \pi \alpha \nu \varepsilon \iota \rho \tilde{\eta} \sigma \vartheta \alpha \iota$ und liefert noch manche sichere Er-

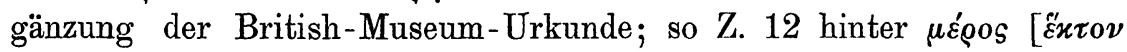

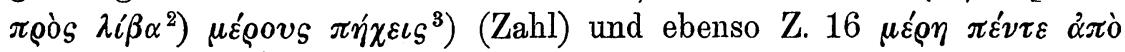

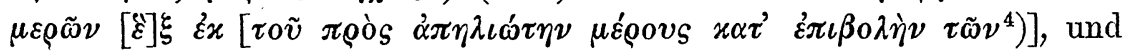
anderes, auf das ich noch komme.

1) Ausführlicher legte ich dies dar: Berl. Philol. Wochenschrift 1899, S. 1571:

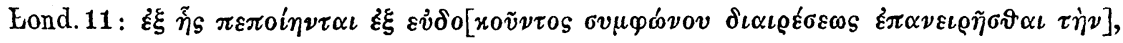

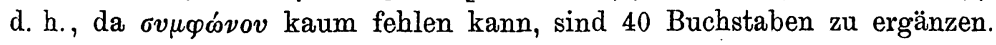

2) Oder die umgekehrte Windrichtung.

3) Zu $\pi \eta^{\prime} \chi \varepsilon \iota s$ vgl. Lond. II 154 (II, S. 179).

4) $\mathrm{Zu}$ dem Schluf's vgl. BGU 444, 19.

Archiv f. Papyrusforschung. I. 1. 
Von nichtjuristischen Worten sind selten und erheblich $\mu \varepsilon \dot{\varepsilon} \tau \rho o \nu$ - $\pi \tilde{\eta} \chi v_{S}-\vartheta \tilde{v} \rho \alpha$; sie finden sich im Lond. II alle drei vereint in CCIV, 6 (S. 179), und diese Nummer bietet mit $\mu \varepsilon \varepsilon \rho o \nu$ und $\pi \tilde{\eta} \chi v_{S}$ nicht nur den Schlufs von Z. 12 (s. oben), sondern in Z. 6/7 auch die Er-

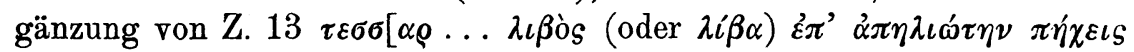
(Zahl)], während wieder das erhaltene vó Papyrus die Ergänzung dort Z. 6 und $7 \varepsilon\left[i_{s}\right]$ zu gunsten von $\varepsilon[\pi i]$ be-

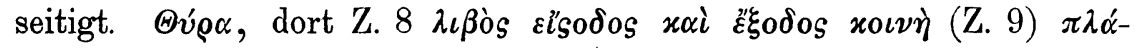

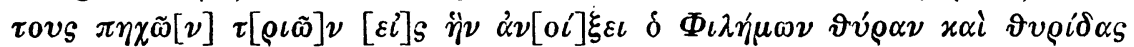
auf unsere Urkunde von Z. 18 übertragen, bildet einen möglichen Schlufs von Z. 17, während (dort Z. 9) die Fortsetzung zur Ergänzung von

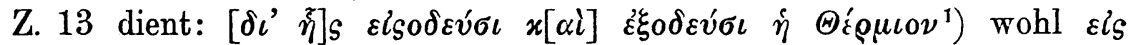
........ BGU 444, 14 lehrt uns noch, dals Z. 15 „der Anspruch" der

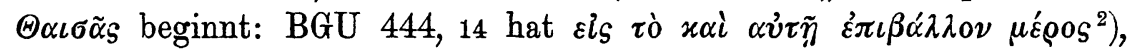

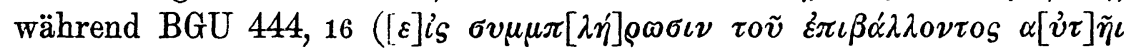

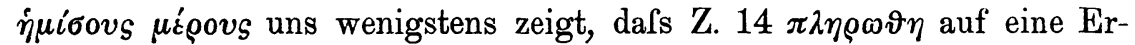
gänzung des Teils der @é́

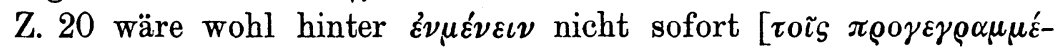

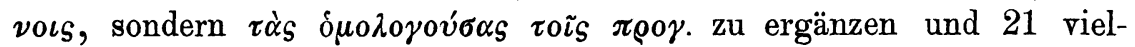

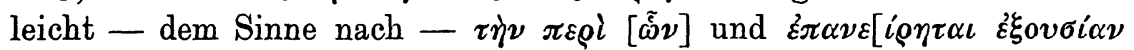

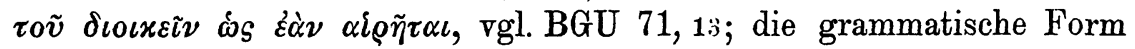
finde ich nicht. ${ }^{3}$ )

Der Schlufs ist mit Hilfe des Wortes $\dot{\pi} \pi$ ่ $\varepsilon^{\prime} \gamma \varrho \alpha[\mu] \mu \varepsilon$ herzustellen: Kenyon führt uns durch seine Lesung 179, 25 (S. 208) und die An-

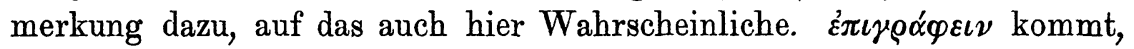
abgesehen von zweifelhaften Stellen ${ }^{4}$ ), in zwei Bedeutungen vor: 'zuschreiben' und 'drüberschreiben', nämlich 'bestellen' und 'fälschen'.

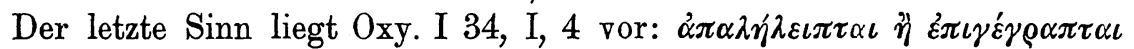

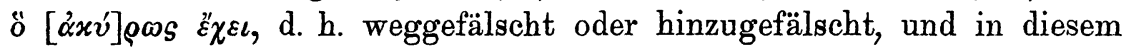
Sinne ist $\dot{\varepsilon} \iota \iota \gamma \rho \alpha \varphi \eta^{\prime}$ und $\dot{\alpha} \lambda \varepsilon \iota \varphi \alpha^{\prime} s$ technisch. - Alle übrigen Stellen geben die zweite Bedeutung in der Weise, die Oxy. I 56, (12) 16 ff. klar-

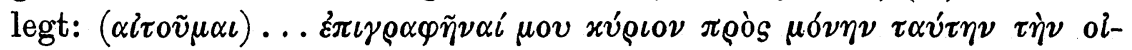

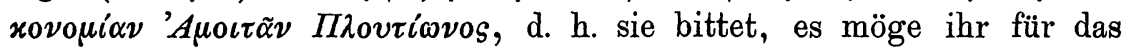
eine negotium (in scriptis habendum) ein xú

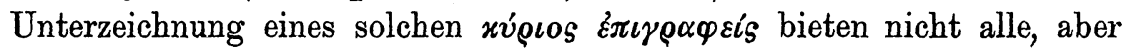

1) $0 \operatorname{der} \Theta \alpha \iota \sigma \tilde{\alpha} s$ ?

2) Indefs ist $x \alpha i$. statt $x \alpha \mu$. zu lesen; vielleicht $x \alpha \iota \quad \alpha[\dot{v} \tau \eta \dot{\nu} \quad \xi \pi \alpha v \varepsilon \iota \rho \tilde{\eta} \sigma \vartheta \alpha \iota \tau \dot{\alpha}$

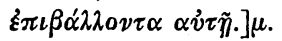

3) Z. 28 ist wohl $\delta \mu \circ \lambda \circ \gamma \circ \tilde{v} \mu \varepsilon \nu$ $\delta \iota \varepsilon \iota \varrho \tilde{\sigma} \sigma \vartheta \alpha \iota$ statt $\delta \iota \varepsilon i \varrho \eta \nu \tau \alpha \iota$ zu lesen und zu

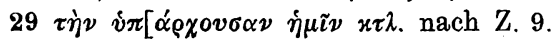

4) BGU 293,$13 ; 457,2 ; 563,8$ und Lond. II $408,10$. 


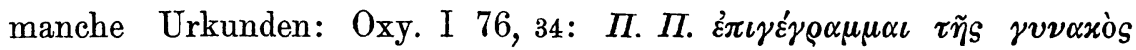

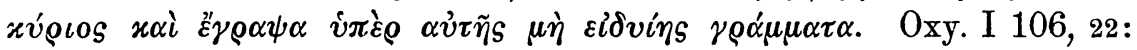

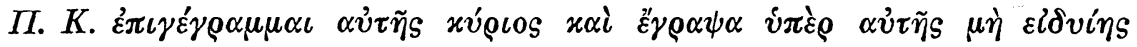

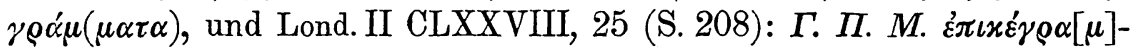
$\mu \alpha \iota$ xv́

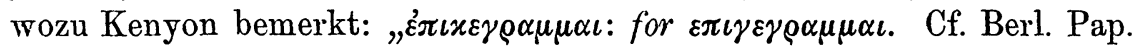

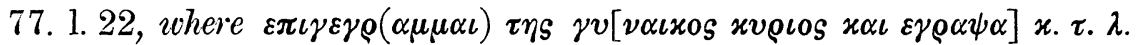

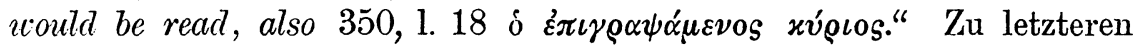

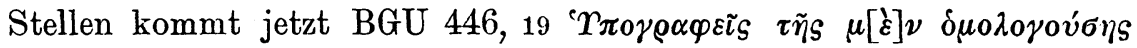

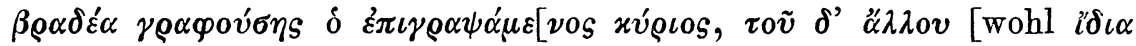

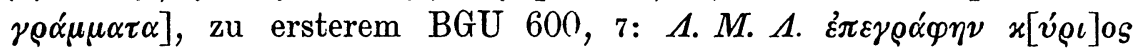

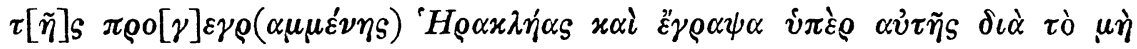

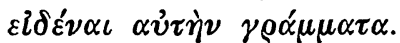

Es ist klar, dafs in unserem Falle nur die xv́oıos-Formel in Betracht kommt, bei dieser sind zwei Abarten zu unterscheiden; einmal wird am Schlufs der Haupturkunde vor der Unterschrift erwähnt, dafs

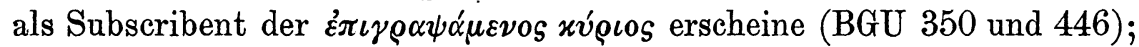
das andremal bemerkt in der Subscriptio der, welcher sie leistet, dafs er zum xúoıos bestellt ward. Beidemal sind aber zu scheiden das

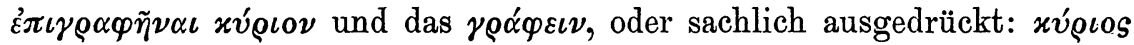

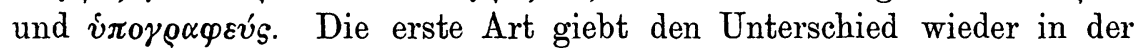

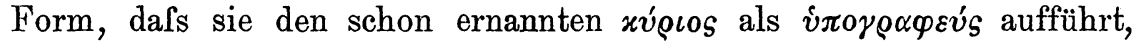
die zweite so, dals der als ernannt sich einführende nachher sich auch

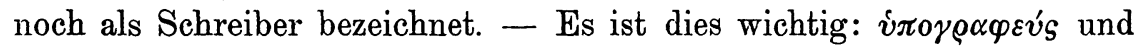

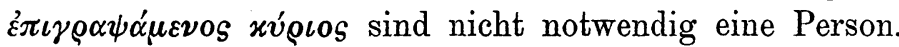

Wenn nun in unserer Urkunde die dritte Hand also erhalten ist

Z. $30 \gamma \varepsilon[\gamma \varrho \alpha \pi \tau \alpha \iota . . . . .].\left[3^{\text {te }}\right.$ Hand.....]

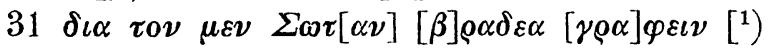

$32 \Sigma \varepsilon \rho \alpha \pi \alpha \tau o s \quad \varepsilon \pi เ \gamma \varepsilon \gamma \rho \alpha[\mu] \mu \varepsilon \quad \tau \eta s[$

$33 x \alpha \vartheta \circ \pi_{0} \pi[x] \iota \tau \alpha \iota$. (4te $4^{\text {te }}$ Hand $\left.)^{2}\right) \varepsilon \tau[o v s] \overline{i 5} \alpha v \tau[0 x \rho \alpha \tau o \varrho o s$

$34 \mu^{\eta} K \alpha \iota \sigma \alpha \rho \varepsilon\llcorner 0 v \overline{x \vartheta}(x . \tau . \lambda$.),

so wird man bei $\tau \tilde{\eta} s$ (Z. 32) an die Frau zu denken haben, die mit dem

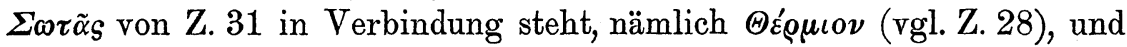
eben diesen, nach Z. 31 schreibungewandten xv́@ıos von demjenigen $\dot{v} \pi 0^{-}$

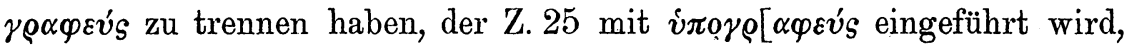

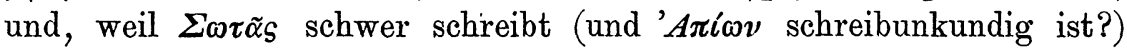
in $2^{\text {ter }}$ Hand das Resumé der $1^{\text {ten }}$ Hand für beide Ausstellerinnen liefert. Bei dem Namen $\Sigma \varepsilon \rho \alpha \pi \alpha \tau o s$ ist zu bedenken, dafs wir von dem xv́@ı⿻

1) Diese Zeile nach dem Faksimile.

2) Z. 33 von hieran nach dem Faksimile. 


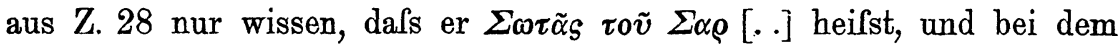
Schreibschwächling ${ }^{1}$ ) der $3^{\text {ten }}$ Hand kann dies sehr wohl zu $\Sigma \varepsilon \rho \alpha \pi \tilde{\alpha} \tau o s$ geworden sein. Hiernach würde ich der $3^{\text {ten }}$ Hand von Z. 31 nur $\Sigma \omega$ $\left.\tau \tilde{\alpha}_{s}\right]$ vindicieren und in Z. 31 die 40-50 Buchstaben zwischen der Schwiegermutterschaft der $\Theta \varepsilon ́ \rho \mu \iota \nu$ und $x \alpha i$ हैy $\rho \alpha \psi \alpha$ (alles $2^{\text {te }}$ Hand) teilen.

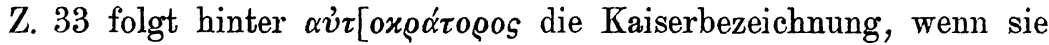
so ausführlich ist wie Z. 1, mit 56 Buchstaben in der 'small, rapid cursive', welche 'the clerk's subscription' hier darstellt.

Man kann hiervon ausgehend auch die letzte Zeile $1^{\text {te }}$ Hand und die $2^{\text {te }}$ Hand noch behandeln. Treffend ergänzt Kenyon $x \alpha \iota \mu \eta \delta \varepsilon \nu ~ \eta \sigma \sigma o \nu$ vor $\tau \alpha \delta_{\iota}[\omega] \mu 0 \lambda \sigma \gamma \eta \mu \varepsilon \nu \alpha$; geht man nun alle Stellen mit $\delta \iota \mu_{0} \lambda \sigma \varepsilon^{\prime} \omega$ durch, so ergiebt sich:

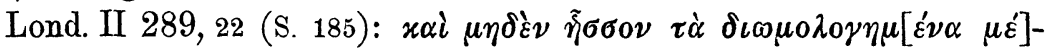

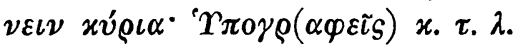

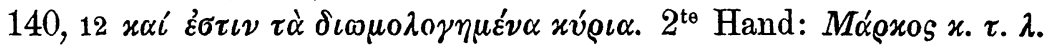

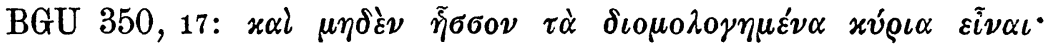
$\Upsilon_{\pi \circ \rho \alpha \varphi \varepsilon \tilde{S}} \varkappa . \tau . \lambda$.

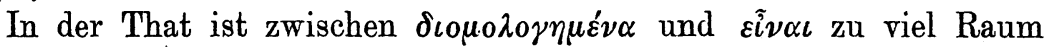
für $[\mu]$, dagegen genügend für $x v ́ \rho \iota \alpha$, und die verwischten Reste gestatten es, diese Buchstaben einzusetzen. Ferner ist zu beachten, dafs

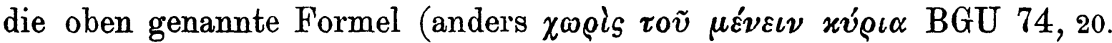
193 , 28. 542, 18. 667, 18) unmittelbar vor dem Aufhören der $1^{\text {ten }}$ Hand

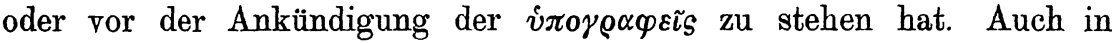

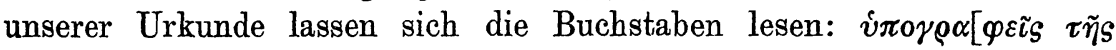

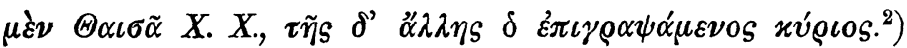

Absichtlich habe ich eine Urkunde, die ein Editor von europäischem Rufe herausgegeben hat, gewählt, um zu zeigen, wie viel auch da noch durch Ausnutzung der Indices mit leichter Mühe sich gewinnen lälst, denn die Inedita, die an die verschiedensten Institute jetzt abgeliefert sind und demnächst publiziert werden, sollen von vornherein sich dies Instrument, das ihre Vorgänger geschaffen haben, zu Nutze machen. Eine ganz andere Frage ist es, ob der Editor gut thut, Ergänzungen, die er zeilenweise durch Analoga findet, buchstäblich hinzuschreiben, oder ob er sie nur zitieren soll. - Der Index ist nicht nur

1) 'in a shaky, irregular, uncial hand'.

2) Z. 19 liefsen die mir zur Verfügung stehenden Indices im Stich. Es heifst am Schlufs nicht $\tau \alpha \iota \nu \tau \eta$, sondern $\tau \omega \nu \tau \eta ; \tau \eta$ ist offenbar der Genitiv, der in $\xi v \lambda \iota x \eta \tilde{s}$ $x \alpha l$ seinen Schlufs findet, und jeder Teilhaberin ihr Teil an den Pertinenzen und am instrumentum fundi sichert; also entweder ist $\tau \tilde{\omega} \nu \tau \tilde{\eta}[s$ zu lesen oder ein mir unbekanntes Wort mit $\tau \eta$ zu ergänzen. 
für Ergänzungen wertvoll, sondern selbst für die Entzifferung schwieriger Stellen: aber da mufs man schon seines Auges sicher sein, dals es einem nicht die fata morgana des erwünschten Simile vorspiegelt.

\section{Stellenverzeichnis und Wortverzeichnis; Konträr-Index.}

Ist von einem Wort nur ein Teil der Buchstaben erhalten, so ist es an sich nicht notwendig, dafs die fehlenden das Vorhandene zu irgend einem bereits bekannten Worte ergänzen, denn die Papyri bringen viele neue Wörter ans Tageslicht; aber es ist wahrscheinlich, dafs das betreffende Wort im Lexikon, und namentlich in den Papyrus-Indices bereits enthalten ist. Die meisten Worte wird der Kenner der Papyri aus dem Gedächtnis rekonstruieren; wo diese Rekonstruktion, die ihre subjektiven und ihre objektiven Erfordernisse hat, nicht gelingt, bietet der Index ein Hilfsmittel für den Fall, dals der Anfang erhalten ist; denn in seinen Lemmata giebt er, wenn z. B. $\alpha \pi$ erhalten, die Möglichkeit, alle Worte mit $\alpha \pi$ der Reihe nach zur Kenntnis zu nehmen, und Erwägungen über das zu Ergänzende anzustellen. Hierzu braucht man offenbar die Stellen zunächst nicht, sondern eben nur die Lemmata, die Stellen hindern die Übersicht; aber doch stören sie nicht so sehr, dafs es sich notwendig machte, die Worte noch einmal zu diesem Zweck ohne Stellenzahlen abzudrucken. Ist aber ein Teil aus der Mitte erhalten, so kann man den Index nur dann gebrauchen, wenn aus sachlichen Gründen nur eine bestimmte Zahl von Worten in Frage kommt:

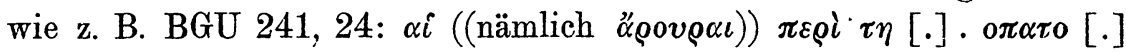

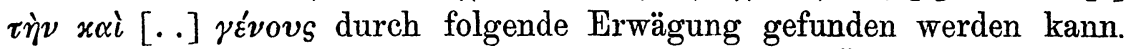
Da es sich um Aruren handelt, so ist mit $\pi \varepsilon \rho i$ die Örtlichkeit, wo sie liegen, eingeführt; daher wird sich die gewünschte Ergänzung unter den Dorfnamen befinden; sieht man diese im Index BGUI VII B durch, so findet sich eine einzige, die die Lettern ... олкло ... in der Mitte ihres Namens birgt, nämlich $\Phi\llcorner\lambda]$ o $\alpha_{\alpha} \tau o\left[\rho_{5}\right.$; Kombinationen, die ich an anderem Orte ausführe, bringen für die weiteren Worte auf $\tau \dot{\nu} \nu x \alpha i$ $\Theta[\varepsilon 0] \gamma \varepsilon \dot{v o v}$; aber den ersten und wichtigsten Namen liefert die einfache Durchsicht der Dorfnamen. - Natürlich kann dies nur bei SpezialIndices geschehen: niemandem kann zugemutet werden, einen GeneralIndex ganz durchzusehen, um einige Mittelbuchstaben zu rekonstruieren. Auch wenn der Schlufs eines Wortes erhalten ist, leisten die vorhandenen Indices nicht den gewünschten Dienst: BGU 667, 16 steht $\dot{\alpha} \nu \varepsilon \mu[\ldots \ldots$; Krebs ergänzt mit Recht $\dot{\alpha} \nu \varepsilon \mu[\pi \circ \delta i \sigma \tau \omega s]$, wahrscheinlich aus dem Gedächtnis; indefs konnte auch ein minder Geübter darauf kommen, wenn er sich durch den Index überzeugte, dals $\alpha \nu \varepsilon \mu \pi \delta_{0} i \sigma \tau \omega s$ 
das einzige Wort mit $\alpha \dot{\nu} \varepsilon \mu$ in den Papyri (die Winde von Oxy. I 100, 10 kommen nicht in Betracht); aber wenn es in derselben Zeile zu An-

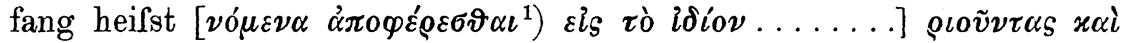

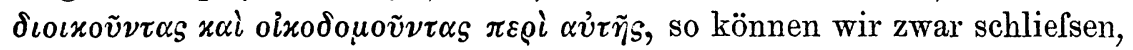

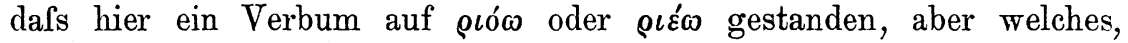
können wir durch kein Wortverzeichnis finden, sondern nur, wenn das Glück gut ist, durch ein Stellenverzeichnis, auf Grund dessen wir die

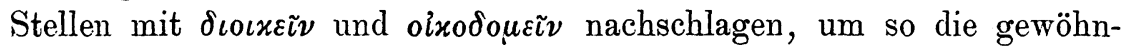
liche Nachbarschaft dieser Worte durchzuprüfen. Allein hier läfst sich Abhilfe schaffen. Sowie nämlich unsere Indices die Worte nach dem Anfang, dem Vorderende, ordnen, kann man sie auch nach dem Schlufsbuchstaben und so fort ordnen: hat man sie in diesem Sinne alphabetisch geordnet vor sich, so kann man für das Vorderende ebenso sicher die Möglichkeiten erwägen, wie beim gewöhnlichen Wörterverzeichnis für den Schlufs. - Ein solches Verzeichnis nach der Ordnung der Wortenden habe ich für die Indices zu BGU I und II., Lond. II, und Oxy. I zu-

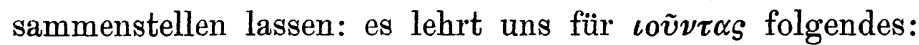

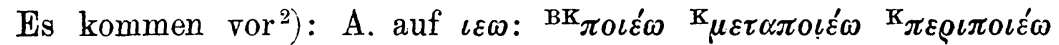

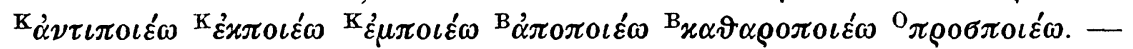

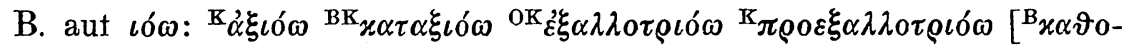

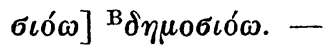

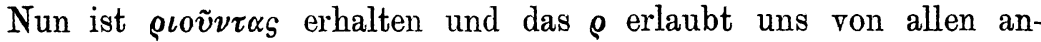

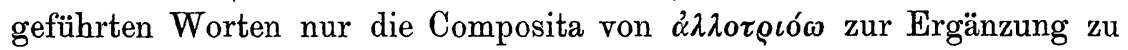

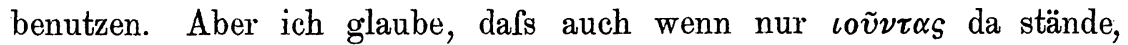
unsere Wahl doch nur auf diese gefallen wäre. Verifiziert man die

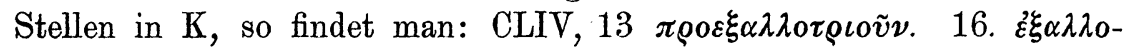

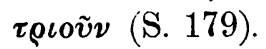

CLIV ist Verkauf einer $\alpha \dot{v} \lambda \dot{\eta}$, BGU 667 Verkauf einer oixi $\alpha$, und in beiden findet sich die stereotype Entäufserungserklärung mit dem Anhang, niemand solle den Erwerber daran hindern, auf dem Grundstück als Herr zu schalten ( $x$ $\varrho \varepsilon v \varepsilon \varepsilon v$ ), was dann spezialisiert wird, und hierbei bringt CLIV eben auch $\tilde{\varepsilon} \xi \alpha \lambda \lambda$ ov

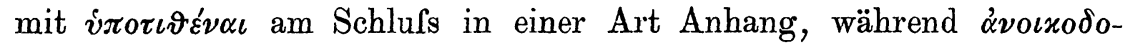

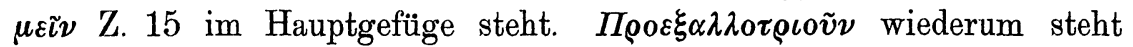

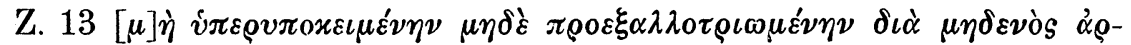
$\chi \varepsilon i \varphi_{0} v$, ähnlich wie Oxy. I 100, 11 (auch ein Grundstückskauf) sagt: siv $\alpha i$

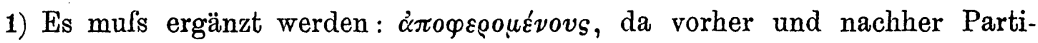
cipia stehen.

2) Die Exponenten BOK beziehen sich auf das Vorkommen des betr. Wortes in den Indices und zwar ist: $\mathrm{B}=\mathrm{BGU}, \mathrm{K}=$ Kenyon II (Lond. II); $\mathrm{O}=$ Oxyrhynchos I. 


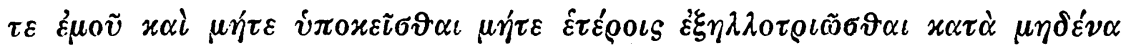

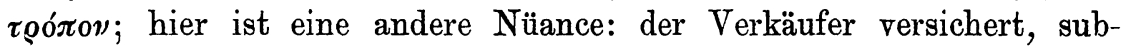
jektiv weder vorverpfändet noch (vor-)veräufsert zu haben, während er Lond. II. CLIV sich für die objektive Pfandfreiheit verbürgt.

Es mufs dem Skeptiker überlassen bleiben, ob er die Ergänzung

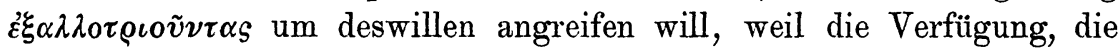

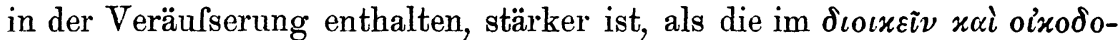
$\mu \varepsilon i \nu$ gelegene, und sie also streng genommen an den Schlufs gehört. Für derartige Indices nach dem Wortende schlage ich die Bezeichnung Conträr-Indices vor; es leuchtet ein, dafs ein Conträr-Index nicht nur für die Zwecke der Ergänzung von Wert ist, sondern auch für linguistische und grammatische: Der gewöhnliche, normale, Index zeigt uns alle Worte, die mit einer bestimmten Präposition, z. B. $\alpha \dot{\pi} \delta$, anfangen; der Conträr-Index ungekehrt giebt uns alle Composita eines bestimmten Verbi, wie das oben für $\pi$ o $\varepsilon^{\prime} \omega$ in den drei Sammlungen dargelegt wurde; der normale Index zeigt uns die Worte, die von einem Stamm, z. B. $\xi v \lambda$ - gebildet werden, der Conträr-Index giebt uns die Stämme aus $v \lambda$-, wenn wir die Suffixa abziehen. Schwierigkeiten macht die Frage der Media, der Pluralia tantum, der substantivierten Adjektiva: man wird im Zweifel beide Formen zu geben sich entschliefsen müssen.

Natürlich sind entfernt nicht alle lexikalischen Beziehungen hiermit erschöpft; doch mögen diese Bemerkungen einstweilen genügen, vielleicht folgt ihnen später ein Wörterbuch-Artikel, etwa über eine Präposition, ist doch gerade bei diesen eine Übersicht über alle Stellen besonders wertvoll.

Königsberg.

0tto Gradenwitz. 